\title{
MedienPädagogik
}

Zeitschrift für Theorie und Praxis der Medienbildung

\section{Editorial: Frühe Medienbildung}

Dorothee M. Meister, Henrike Friedrichs und Uwe Sander

Frühkindliche Bildung hat sich an der konkreten und aktuellen Lebenswirklichkeit von Kindern zu orientieren. Und ein markantes Charakteristikum der heutigen Kindheit ist ihre Eigenschaft als Medienkindheit. Das gilt auch schon für Kinder im Vorschulalter, die sowohl als Medienrezipienten als auch als Warenkonsumenten fungieren und in beiderlei Hinsicht eine relevante Zielgruppe darstellen (vgl. PausHasebrink und Kulterer 2014; Egmont Ehapa 2013). Als pädagogische, aber auch bildungspolitische Reaktion auf diese Mediatisierung der Kindheit mit den damit einhergehenden Anforderungen und Verlockungen gewinnt gegenwärtig Medienkompetenz und -bildung einen immer gewichtigeren Stellenwert in der frühkindlichen Bildung und Erziehung. Kinder, so die Forderung, sollen in der technisierten Wissensgesellschaft Medien eigenverantwortlich und selbstverantwortlich handhaben und sie als Wissens- und Entwicklungsressource nutzen können. Das dazu notwendige Wissen und Können erlernen Kinder informell z.B. im «ökologischen Zentrum» (Baacke 1999), dem Elternhaus, und zunehmend auch in der Gleichaltrigengruppe. Allerdings garantieren diese Lebenskontexte von Kindern nicht für alle eine hinreichende Vorbereitung auf die Medienwelt, z.B. weil die Kovarianz von sozialer Ungleichheit und Bildungsbenachteiligung auch für Medienbildung gilt. Im Hinblick auf systematisches und strukturiertes Lernen vor der Schule geraten in der Folge Kindertagesstätten konsequent in den Fokus einer öffentlich zu sichernden Medienbildung in der Phase der Vorschulkindheit. Diese ansonsten eher fürsorglichen und erzieherischen, statt qualifizierenden Einrichtungen der frühkindlichen Bildung sollen medienpädagogisch aktiv werden und, was ein Novum darstellt oder sogar auf Ressentiments stößt, auch auf die Nutzung der Welt neuer und digital-vernetzter Medien vorbereiten. Im Rahmen der Professionalisierung der Frühpädagogik und des damit einhergehend gestärkten Bildungsauftrags der Kindertagesstätten wird innerhalb der Bildungspläne der Bundesländer neben weiteren Bildungsaufgaben eine solche Erwartungshaltung an ErzieherInnen formuliert (vgl. Friedrichs und Meister 2015; Neuß 2013). Da der Bereich der Medienbildung jedoch nach wie vor einen eher geringen Stellenwert in den vorschulischen Einrichtungen einnimmt (vgl. Institut für Demoskopie Allensbach 2015), werden ErzieherInnen trotz solcher Forderungen realiter eher punktuell und in Pilotprojekten fortgebildet. Der bislang eher medienabstinente bis medienfeindliche Sektor der frühkindlichen Bildung scheint sich also faktisch in der Bundesrepublik Deutschland nur langsam in Richtung professioneller Medienkompetenzförderung zu bewegen. 
Die Beiträge dieses Themenhefts schließen an die oben angerissene Diskussion an. In ihnen werden verschiedene Aspekte um Kindheit und Medien(bildung) beleuchtet und basierend auf empirischen Studien das Medienerziehungsverhalten im Elternhaus und die damit einhergehende kindliche Medienaneignung betrachtet. Darüber hinaus werden der Erfolg medienpädagogischer Fortbildungen, beispielsweise hinsichtlich einer notwendigen Offenheit von ErzieherInnen für die Thematik der Medienbildung, in den Blick genommen und Schlüsse für eine nachhaltige Verankerung von Medienbildung im schulischen Bereich gezogen.

Folgende Beiträge liegen vor:

Nadia Kutscher diskutiert in ihrem Beitrag die Ziele und Schwerpunkte von Medienbildung in der Kindheit sowie die damit verbundene Adressierung von Familie und Kindheit im Kontext von Mediennutzung und -erziehung. Zunächst zeigt die Autorin verschiedene Diskurse um Medien und Kindheit auf und verweist mit Blick auf die Ergebnisse der KIM-, JIM- und FIM-Studien (mpfs) auf die mediatisierte Lebenswelt von Kindern. Anschließend richtet sie ihren Blick auf die Bildungspläne der Bundesländer und die darin formulierte Aufgabe einer Förderung der kindlichen Medienkompetenz, welche die Autorin als Teil eines Kompetenzkanons betrachtet, den Kinder sich in (vor-)schulischen Einrichtungen aneignen sollen.

Burkhard Fuhs beleuchtet in seinem Beitrag den Bildungsbereich der Medienpädagogik in der frühen Kindheit. Aktuell sei der Diskurs um Medienbildung eher defizitär ausgerichtet, indem ein intensiver Mediengebrauch als Gefahr für Kinder betrachtet werde. Fuhs fordert angesichts der medialen Durchdringung der frühen Kindheit, Medien stärker in die pädagogische Arbeit vorschulischer Einrichtungen zu integrieren. Dabei müssten die besonderen Anforderungen dieses pädagogischen Bereichs berücksichtigt werden.

Christian Swertz, Gudrun Kern und Erika Kovacova stellen die Ergebnisse eines Forschungsprojekts zur Medienkultur 3- bis 6-jähriger Kinder vor. Den theoretischen Referenzrahmen der Studie bildet das in Anlehnung an Bourdieu entwickelte Konzept des medialen Habitus. Der mediale Habitus wird von den Autorlnnen als «Ausdruck des von Menschen bewusst und unbewusst wahrgenommenen Eindrucks, den Medien im Menschen hinterlassen» verstanden. Ein zentrales Ergebnis des Forschungsprojekts ist, dass Unterschiede im Ausdruck des medialen Habitus der Kinder vor allem auf das kulturelle Kapital der Eltern und weniger auf das verfügbare ökonomische Kapital zurückzuführen sind. 
Marion Weise geht in ihrer qualitativen Studie, die auf 21 qualitativen Interviews mit Kindern aus Deutschland und den USA basiert, der Frage nach, inwiefern Kinder unterschiedliche Verantwortlichkeiten für Medienerziehung und Medienreglementierungen in ihren Familien wahrnehmen. Die Rekonstruktion der qualitativen Daten zeigt, dass die Kinder der vorliegenden Studie ihre Mütter als Kontrollinstanzen der kindlichen Mediennutzung verstehen, die Väter hingegen zu Medienverbündeten der Kinder werden können. Darüber hinaus werden die Väter im Vergleich zu den Müttern von den befragten Kindern als medienaffiner ausgewiesen und fungieren überwiegend als die kompetenten Ansprechpartner für Medientechnologien. Dementsprechend resümiert Weise mit Blick auf die Analyseergebnisse, dass Kinder geschlechtsbezogene Unterschiede im Hinblick auf die elterliche Medienverantwortung sehen.

Gudrun Marci-Boehncke, Matthias Rath und Anita Müller haben 21 Kindertagesstätten im Dortmunder Norden, die über die Initiative "KidSmart» mit Computern ausgestattet und deren ErzieherInnen medienpädagogisch fortgebildet wurden, in einer Forschungs- und Interventionsstudie vor dem Hintergrund der Kapitalsortenund Habitustheorie Bourdieus in den Blick genommen. In diesem Rahmen wurden sowohl Erzieherlnnen zu ihrer eigenen Mediennutzung und -kompetenz als auch ErzieherInnen, Eltern und Kita-Kinder zum Medienverhalten der Kinder befragt. Die Ergebnisse der Studie aus dem ersten Projektjahr zeigen u.a. eine Zunahme technischer Kompetenzen und eine Abnahme bewahrpädagogischer Einstellungen aufseiten der ErzieherInnen auf. Zudem sehen ErzieherInnen und Eltern einen Anstieg technischer, kognitiver und sozialer Kompetenzen der Kinder.

Henrike Friedrichs und Dorothee M. Meister stellen das Fortbildungskonzept der medienpädagogischen Initiative «Medienkompetenz-Kitas NRW» vor, das mit einem niederschwelligen Inhouse-Schulungsansatz mit zwölf Kindertagesstätten in NRW durchgeführt wurde. Ziel des Projekts war die Sensibilisierung und Aktivierung der Kita-Mitarbeiterinnen für den Bereich Medienerziehung, um Medienerziehung praxisnah und nachhaltig an den Einrichtungen zu verankern. Im Fokus standen die Vermittlung technischen Grundlagenwissens, handlungsorientierte Methoden für eine aktive medienpraktische Arbeit sowie medienpädagogischen Reflexionswissens durch Medien-Coaches. Auf der Grundlage einer komplexen Evaluation des Projektes werden weiterführende Überlegungen zur Nachhaltigkeit medienpädagogischer Fortbildungen im Vorschulbereich vorgestellt. 


\section{Literatur}

Baacke, Dieter. 1999. Die 0- bis 5jährigen: Einführung in die Probleme der frühen Kindheit. Weinheim und Basel: Beltz.

Egmont Ehapa. 2013. KidsVerbraucherAnalyse 2013. Berlin.

Friedrichs, Henrike und Dorothee M. Meister. 2015. «Medienerziehung in der Kindertagesstätte.» In Medienpädagogik - ein Überblick, hrsg. v. Friederike von Gross, Dorothee M. Meister u. Uwe Sander, 273-305. Weinheim und Basel: Beltz Juventa.

Institut für Demoskopie Allensbach. 2015. «Digitale Medienbildung in Grundschule und Kindergarten: Ergebnisse einer Befragung von Eltern, Lehrkräften an Grundschulen und Erzieher(innen) in Kindergärten im Auftrag der Deutsche Telekom Stiftung.» http://www. telekom-stiftung.de/dts-cms/sites/default/files//dts-library/materialien/pdf/ergebnisse_ allensbach-umfrage_gesamt.pdf.

Neuß, Norbert. 2013. «Medienkompetenz in der frühen Kindheit.»In Medienkompetenzförderung für Kinder und Jugendliche: Eine Bestandsaufnahme, hrsg. v. Bundesministerium für Familie, Senioren, Frauen und Jugend, 34-45. Berlin.

Paus-Hasebrink, Ingrid und Jasmin Kulterer. 2014. "Kommerzialisierung von Kindheit.»In Handbuch Kinder und Medien, hrsg. v. Angela Tillmann, Sandra Fleischer u. Kai-Uwe Hugger, 47-57. Wiesbaden: Springer VS. 